\title{
SOX9 in prostate cancer is upregulated by cancer- associated fibroblasts to promote tumor progression through HGF/c-Met-FRA1 signaling
}

\section{Haixiang Qin}

Nanjing University Medical School Affiliated Nanjing Drum Tower Hospital

\section{Yang Yang}

Nanjing University Medical School Affiliated Nanjing Drum Tower Hospital

Bo Jiang

Nanjing University Medical School Affiliated Nanjing Drum Tower Hospital

\section{Chun Pan}

Nanjing University Medical School

\section{Wei Chen}

Nanjing University Medical School Affiliated Nanjing Drum Tower Hospital

\section{Wenli Diao}

Nanjing University Medical School Affiliated Nanjing Drum Tower Hospital

\section{Meng Ding}

Nanjing University Medical School Affiliated Nanjing Drum Tower Hospital

\section{Wenmin Cao}

Nanjing University Medical School Affiliated Nanjing Drum Tower Hospital

Zhenxing Zhang

Wannan Medical College

\section{Haoxing Ma}

Shaanxi Provincial People's Hospital

\section{Mengxia Chen}

Nanjing University Medical School Affiliated Nanjing Drum Tower Hospital Jie Gao

Nanjing University Medical School Affiliated Nanjing Drum Tower Hospital

Xiaozhi Zhao

Nanjing University Medical School Affiliated Nanjing Drum Tower Hospital

Xuefeng Qiu ( $\square$ Xuefeng_Qiu@163.com )

Nanjing University Medical School Affiliated Nanjing Drum Tower Hospital

Hongqian Guo ( $\nabla$ dr.ghq@nju.edu.cn )

Nanjing University Medical School Affiliated Nanjing Drum Tower Hospital 
Research

Keywords: SOX9, prostate cancer, cancer-associated fibroblasts, hepatocyte growth factor, FRA1

Posted Date: June 7th, 2020

DOl: https://doi.org/10.21203/rs.3.rs-32822/v1

License: (c) (i) This work is licensed under a Creative Commons Attribution 4.0 International License. Read Full License

Version of Record: A version of this preprint was published at The FEBS Journal on April 2nd, 2021. See the published version at https://doi.org/10.1111/febs.15816. 


\section{Abstract \\ Background}

Previous studies have demonstrated that transcription factor SOX9 which was reactivated in prostate cancer (Pca) and promoted tumor growth was a poor prognostic biomarker for Pca. Nevertheless, the regulatory mechanism underlying SOX9 upregulation in Pca still remains unclear. Several cytokines widely distributed in the tumor microenvironment (TME) have been reported to be involved in the regulation of SOX9, suggesting that cancer-associated fibroblasts (CAFs), one of the main sources of secreted factors in TME, may play a role in regulating SOX9 expression.

\section{Methods}

Herein, an in vitro model of paracrine interaction between primary CAFs and Pca cells (both AR-positive and AR-negative Pca cells), was applied to investigate the molecular mechanism of SOX9 upregulation during Pca progression. The regulatory axis was validated by in vivo experiments and The Cancer Genome Atlas (TCGA) data.

\section{Results}

Conditional medium from Pca CAFs (CAF-CM) upregulated the expression of SOX9, which was also proved to be essential for CAF-induced tumor progression. Further analysis showed that it was hepatocyte growth factor (HGF) secreted by CAFs that was responsible for the SOX9 elevation in Pca cells via activating c-Met signaling. Mechanistically, HGF/c-Met signaling specifically activated MEK1/2ERK1/2 pathway which then induced phosphorylated status and protein upregulation of FRA1. Furthermore, ChIP assay demonstrated that FRA1 transcriptionally upregulated SOX9 expression by binding to the TPA-responsive element (TRE) sequence in the promoter of SOX9 gene. We also found that HGF/c-Met-ERK1/2-FRA1-SOX9 axis was relatively conserved in human and mouse species by validating in mouse Pca cells (RM-1).

\section{Conclusions}

Our results revealed a novel insight into the molecular mechanism that SOX9 expression in Pca cells is promoted by CAFs, through the HGF/cMet-ERK1/2-FRA1 axis. Besides, SOX9 may serve as an alternative marker for the activated HGF/c-Met signaling to enroll the optimal Pca patients for HGF/c-Met inhibition treatment, since it is much more stable and easier to detect.

\section{Background}


SOX9 is a transcription factor belonging to the SOX (Sry-related high mobility group box) family, which is a key regulator involved in embryonic development including sex determination [1], chondrocyte differentiation [2], neurogenesis-gliogenesis switch [3], cardiogenesis [4], as well as prostate development $[5,6]$. Previous studies showed that SOX9 played an important role in the initial stage of prostate development. Mice with conditional Sox 9 knockout in prostate showed severe defect in the development of ventral prostate and abnormal differentiation of anterior prostate [5]. It is a common occurrence that genes or pathways involved in embryogenesis are reactivated in cancer initiation and development. Several studies have indicated that SOX9 was reactivated in prostate cancer (Pca) and played an essential role in the initiation and progression of Pca [6-9]. Thomsen et al. observed that Sox9 elevation in mouse prostate could facilitate Pca initiation in cooperation with Pten loss [8]. Wang et al. found that upregulation of SOX9 promoted Pca cell proliferation and invasion, as well as angiogenesis, indicating that SOX9 contributed to Pca progression [7]. Further investigations showed that SOX9/Sox9 was expressed at higher frequency in recurrent $\mathrm{Pca}[10]$ and strikingly upregulated in castration-resistant tumors of Pten/Trp53 null mice [11]. When SOX9 was knocked down in androgen-independent Pca cells, it obviously weakened the clonogenicity in castrated culture medium and tumor growth in castrated mice [12].

Despite the significance of SOX9 in Pca, the key regulatory mechanism underlying SOX9 upregulation still remains unclear. A previous study revealed that SOX9 expression in Pca was regulated by $\mathrm{Wnt} / \beta$-catenin signaling. However, the exact mechanism was not further investigated [10]. Cai et al. found that in TMPRSS2:ERG fusion-positive Pca cells, such as VCaP, SOX9 expression could be upregulated by ERG through opening an androgen receptor (AR)-regulated enhancer downstream of the SOX9 gene. However, this mechanism was strictly restricted in the ERG and AR double-positive Pca cells [9].

There has been increasing evidences implying that cytokines such as FGFs $[13,14]$, TGF $\beta 1[15,16]$, BMPs $[17,18]$, are involved in the regulation of SOX9 expression. It is well known that large amounts of cytokines distributed in the tumor microenvironment (TME) play important roles in cellular communications. As one of the most abundant stromal cells in TME, cancer-associated fibroblasts (CAFs) have been demonstrated to promote tumorigenesis and progression of multiple tumors including Pca [19-24] through paracrine effects. Kinds of cytokines secreted by CAFs have been reported to contribute to cancer cell stemness through activating self-renewal pathways including Wnt, Notch and Shh signaling [25-27]. SOX9 is also well known to be involved in maintaining stem cell populations in normal tissues $[10,13,28,29]$ and cancer cell stemness $[12,30]$. In addition, SOX9 ChIP sequencing analysis and transcriptome profiling of Pca cells showed that SOX9 positively activated various Wnt pathway genes to drive invasiveness of Pca [31]. In light of these findings, we hypothesized that SOX9 elevation in Pca cells might be regulated by secreted cytokines from CAFs, and mediate the tumorpromoting effects of CAFs.

This study was therefore designed to investigate the possible mechanism of SOX9 elevation by CAFs through an in vitro model of paracrine interaction between primary CAFs and Pca cells. To the best of our 
knowledge, this is the first study to illustrate the regulatory mechanism of upregulated SOX9 by CAFs during the progression of Pca.

\section{Materials And Methods}

\section{Cell lines and cell culture}

Four human prostate cancer cell lines, LNCaP, 22Rv1, DU145 and PC3, were obtained from the Cell Bank of Type Culture Collection, Chinese Academy of Science (Shanghai, China), and were authenticated by the short tandem repeat analysis (CellcookBiotech, Guangzhou, China) according to the American Type Culture Collect (ATCC) guideline. RWPE-1, derived from a histologically normal adult human prostate, and RM-1, a mouse prostate cancer cell line, were purchased from ATCC.

\section{Clinical Pca tissues and immunohistochemistry $(\mathrm{IHC})$ analyses}

Human Pca specimens for IHC staining, along with corresponding clinicopathologic features, were collected from Nanjing Drum tower Hospital (Nanjing, China) from January 2011 to January 2014 (n $=108$ ), and the IHC staining was performed as described previously [36]. The scores of SOX9 IHC staining were performed independently by two well-trained pathologists. The staining intensity was scored as 0 (negative), 1 (weak), 2 (moderate), 3 (strong), and the staining range of tumor cells was scored as 0 ( $0 \%$ ), 1 (1\%-10\%), 2 (11\%-50\%), 3 (51\%-100\%). The final score was obtained as the multiplication of the intensity score by the staining proportion, and a score $\leq 3$ was defined as low expression or negative, while $>3$ as high expression.

\section{In vivo xenograft tumor model}

Animal experiments were approved and performed in accordance with Institutional Animal Care and Use Committee of Nanjing Drum Tower Hospital (Nanjing, China). Six-week-old male BALB/c nude mice which were purchased from the Animal Core Facility of Nanjing Medical University (Nanjing, China) were housed in the specific pathogen free environment at the Animal Laboratory Center of Nanjing Drum Tower Hospital. In order to determine whether CAFs and HGF play an important role in regulating tumorigenesis and tumor development, $2 \times 10^{6}$ DU145 cells alone or mixed with equivalent CAFs were injected subcutaneously into the right flank of nude mice. 21 days post inoculation, Vehicle or capmatinib (INCB28060) (Selleck Chemicals, S2788, USA) $(10 \mathrm{mg} / \mathrm{kg}$ body weight) , were given to the tumor-bearing mice via orogastric gavage twice a day. Tumor diameters were measured every 3 days and tumor volumes were calculated as length $\times$ width $^{2} \times \pi / 6$. The tumors were harvested 4 hours after the last dosing and snap frozen in liquid nitrogen or fixed in paraformaldehyde. For the purpose of assessing the effects of SOX9 and FRA1 on the proliferation of prostate cancer, $4 \times 10^{6}$ DU145 derived cells were injected into the right flank of nude mice subcutaneously, then tumors were measured every 3 days and collected at the end point for IHC staining.

\section{Statistical analysis}


Continuous normally distributed variables were presented as mean \pm standard deviation (SD), and unpaired Student's t test was used to evaluate the statistical significance between two groups. Categorical data were analyzed by chi-square test or Fisher exact test. Pearson correlation test was applied to analyze the correlation between two continuous normally distributed factors, while Spearman correlation was used to analyze the relationship between categorical variables. All data analysis was performed by using IBM SPSS statistics 21.0 , and $p<0.05$ was considered statistically significant ( ${ }^{\star} p<$ $0.05 ; * \star p<0.01 ; * \star \star x<0.001)$.

Other experimental procedures please refer to the Supplementary Materials and Methods for details.

\section{Results}

\section{SOX9 elevation in Pca is positively correlated with cancer aggressiveness}

In order to verify the clinical significance of SOX9 in human Pca, we firstly examined SOX9 expression in specimens of radical prostatectomy from a cohort of 108 consecutive Pca patients by IHC staining. For pathological analysis, Pca with higher Gleason scores had higher expression of SOX9 (Fig. 1A, B). Particularly, specimens with Gleason scores no less than 8 showed significantly higher expression of SOX9 than $3+3,3+4$ and $4+3$ tissues (Fig. 1B). Moreover, Pca with advanced tumor stage (III and IV) also showed higher SOX9 expression (Fig. 1C). Spearman correlation analysis indicated that SOX9 expression was significantly associated with the percentage of Ki67-positive tumor cells (Spearman $r=0.301, p$ $=0.0017, \mathrm{n}=108$ ) (Fig. 1D). In addition, high SOX9 IHC staining in Pca patients was positively associated with Gleason scores $(p=0.004)$, seminal vesicle invasion $(p=0.040)$ and nerve invasion $(p=0.019)$ (Table S1). These results validated previous reports $[7,8,10]$ that SOX9 levels correlated with Pca progression.

To explore the possible roles of SOX9 in Pca tumorigenesis and progression, we subsequently assessed the effects of SOX9 on proliferation, migration, invasion and stemness in Pca cells. DU145 cells transiently transfected with pcDNA3.1(+)-SOX9 (Fig. S1A, B) showed a remarkably enhanced growth rate compared with the negative control group (Fig. S1C). Transwell assay revealed that forced expression of SOX9 notably accelerated cell migration and invasion of DU145 cells (Fig. S1D, E). Spheroid formation assay indicated that over-expression of SOX9 significantly increased both the size and number of cell spheres (Fig. S1F, G). Consistently, immunoblotting analysis showed that SOX9 overexpression led to the increase of MMP1 (metastasis-associated protein) and CD44, CD133, c-Myc, Oct4, ALDH1A1 (tumor stemness-associated markers) (Fig. S1H, I). Then, another Pca cell line PC3, which had a higher endogenous expression of SOX9, was knocked down by siRNA (siSOX9-1 and siSOX9-2) (Fig. S2A, B). Compared with negative control siRNA ( $\mathrm{SiNC}$ ), in vitro assays revealed that SOX9 interfering markedly inhibited cell growth and viability (Fig. S2C, D), as well as cell migration, invasion (Fig. S2E, F) and cancer stemness (Fig. S2G, H). In addition, decreased protein levels and mRNA expression of CD44, CD133 and c-Myc were also detected in the SOX9 knockdown cells (Fig. S2I, J).

To investigate whether forced expression of SOX9 promote tumor growth of Pca cells in vivo, DU145 cells stably overexpressing SOX9 or vector control (Fig. 1E, F) were injected subcutaneously in nude mice. 
Compared with the control group, xenografts of SOX9 over-expressing cells had a greater tumor volume and growth rate (Fig. 1G, H). Moreover, IHC staining showed that over-expression of SOX9 promoted the expression of Ki67 and CD44, CD133 in DU145 xenograft tumors (Fig. 1I).

\section{SOX9 in Pca is upregulated by CAFs and essential for CAF-induced tumor-promoting effects}

To assess whether SOX9 in Pca cells could be regulated by paracrine factors from CAFs, we isolated CAFs and corresponding normal fibroblasts (NFs) from fresh prostate biopsy specimens (Fig. S3A). Immunofluorescence staining showed that in the biopsy tissues of both normal prostate and prostate cancer, epithelial cells expressed E-cadherin prominently, while stromal cells expressed Fibronectin markedly (Fig. S3B). As for CAF-specific biomarker, a-SMA, was barely detected in the stromal area of normal tissues, while strongly stained in prostate cancer stromal, nearly co-localized with Fibronectin (Fig. S3B). Cell immunofluorescence (Fig. S3C), western blotting and qRT-PCR (Fig. S4A, B) were also performed to verify the purity of cultured fibroblasts. Altogether, the fibroblast identity of NFs and CAFs has been confirmed, and found to be sustaining after several passages (data not shown).

Then, we verified the tumor-promoting effects of CAFs by using an in vitro model of paracrine interaction between primary CAFs and Pca cells. Wound healing assay, transwell assay and spheroid formation assay revealed that treatment with CAF-CM remarkably increased the abilities of cell migration, invasion and cancer stemness (Fig. S5A-F), in comparison with both control medium and conditional medium from NFs (NF-CM). Consistently, western blotting results showed that MMP1 and MMP3, and markers of cancer stemness (CD44, CD133, c-Myc) were elevated in the CAF-CM treated cells (Fig. S5G, H).

To investigate the paracrine effects of CAFs on SOX9 expression in Pca cells, CAF-CM and NF-CM were added to DU145 and 22Rv1 cells for 12 and $24 \mathrm{~h}$. Western blotting and qRT-PCR revealed that both CAF$\mathrm{CM}$ and NF-CM increased SOX9 protein expression and mRNA level, but the effect of CAF-CM was more apparent (Fig. 2A, B). Immunocytochemical staining also demonstrated that since 3 hours after CAF-CM stimulation, SOX9 expression started increasing (Fig. S8J). These results suggested that paracrine factors from stromal fibroblasts upregulated SOX9 expression in Pca cells.

Since CAFs promoted both the aggressiveness of Pca and the expression of SOX9, we wondered whether SOX9 played the role as a bridge in the process of CAF-mediated tumor promotion. RNA interference involving three siRNA targeting different regions was employed to knock down SOX9 expression, then transwell invasion assay and spheroid formation assay showed that depletion of SOX9 significantly reduced the capabilities of cell invasion and sphere formation potentiated by CAF-CM (Fig. 2C-F).

Concomitantly, Western blotting analysis further showed that protein expressions of CD44 and CD133 were reduced in SOX9 knockdown groups compared with siNC cells (Fig. 2G). These data implied that SOX9 played an essential role in the CAF-mediated Pca progression.

HGF is a key paracrine factor secreted by CAFs to mediate SOX9 upregulation in Pca cells 
In order to identify the key paracrine factors secreted by CAFs involved in the activation of SOX9, a homemade qRT-PCR array consisting of 52 TME-related secretory factors was applied to screen in DU145 cells and three pairs of NFs and CAFs for the possibly responsible factors. Hierarchical clustering and heatmap indicated that in the aggregate that genes expressed lower in DU145 cells but highly in fibroblasts, HGF was the most upregulated in CAFs compared with NFs (Fig. 3A). Considering HGF and its tyrosine kinase receptor c-Met are known involved in promoting Pca progression [32, 33], we focused our attention on the role of HGF in CAF-mediated SOX9 upregulation and tumor-promoting effects. Firstly, qRT-PCR results verified that mRNA level of HGF in CAFs was about 13 folds higher than in NFs, while barely detected in DU145 cells (Fig. 3B). ELISA data showed that no or negligible levels of HGF were produced by Pca cells (22Rv1, PC3, DU145), while all CAFs secreted more abundant HGF compared with their paired NFs, suggesting that HGF was preferentially secreted by CAFs in TME (Fig. 3C). Consistently, western blotting analysis confirmed the strikingly increase of c-Met phosphorylation (Tyr1234/1235) in DU145 and 22Rv1 cells following the treatment with CAF-CM compared to NF-CM, while c-Met remained nearly unphosphorylated in control medium (Fig. 3D), which suggested that tyrosine residues 1234 and 1235 of c-Met in Pca cells would not auto-phosphorylated without stimulation of stromal fibroblasts. The correlation between the expression of HGF and ACTA2 (which encodes a-SMA) was evaluated by Pearson correlation analysis using mRNA expression profiles of Pca from TCGA, and it turned out that HGF expression was significantly correlated with ACTA2 (Pearson $r=0.406, p<0.0001, n=499$ ) (Fig. 3E).

Previous studies have demonstrated that HGF/c-Met played an important role in tumorigenesis and tumor progression of Pca [32,33]. Herein, we treated DU145 and 22Rv1 cells with recombinant human HGF (Peprotech, 100-39H, Rocky Hill, NJ, USA) in vitro, and the abilities of cell migration, invasion and sphere formation were detected. As shown in Fig. S6, $10 \mathrm{ng} / \mathrm{ml} \mathrm{HGF}$ was sufficient to significantly increase the number of cells migrated or invaded to the down surface of transwell membrane (Fig. S6AD), and enhance the number and size of formed spheroids (Fig. S6E, F). The key role of HGF in the paracrine effects of CAFs was further validated by an ATP-competitive c-Met inhibitor, capmatinib. In vitro assays showed that the inhibition of HGF/c-Met pathway in DU145 and 22Rv1 cells with pre-treatment of $10 \mathrm{nM}$ capmatinib, remarkably abrogated the effects of CAF-CM on the cell migration, invasion and tumor initiating abilities (Fig. S7A-F).

Since HGF has been identified as a key secretory factor in CAF-CM mediating tumor promotion, and SOX9 was also verified essential for Pca progression induced by CAFs, we wonder whether there was a connection between HGF and SOX9 expression. Western blotting analysis demonstrated that treatment with $30 \mathrm{ng} / \mathrm{ml}$ recombinant human HGF obviously upregulated SOX9 expression since 1 hour upon stimulation in both DU145 and 22Rv1 cells (Fig. 3F). $10 \mathrm{ng} / \mathrm{ml} \mathrm{HGF}$ was already sufficient to induce the increase of SOX9 expression, as well as $30 \mathrm{ng} / \mathrm{ml} \mathrm{HGF} \mathrm{(Fig.} \mathrm{3G).} \mathrm{When} \mathrm{capmatinib} \mathrm{was} \mathrm{combined} \mathrm{in} \mathrm{the}$ HGF or CAF-CM treatment, the upregulation of SOX9 caused by HGF or CAF-CM was markedly abrogated (Fig. 3G, Fig. S8A, B). In addition, in vivo experiments were performed to further investigate the comprehensive role of HGF/c-Met signaling in the regulation of tumor growth and SOX9 expression. DU145 cells alone or companied with equivalent CAFs were injected subcutaneously into nude mice, and three weeks later, vehicle or capmatinib were given to the tumor-bearing mice twice a day. Compared with 
the DU145 alone group, tumors of DU145 with CAFs had a more rapid growth rate and greater tumor size, while capmatinib treatment significantly abrogated the CAF-mediated acceleration of tumor growth (Fig. $3 \mathrm{H}, \mathrm{I})$. IHC staining demonstrated that SOX9 expression in Pca cells was upregulated by CAFs in vivo, and could be restored by capmatinib which strongly inhibited the phosphorylation of c-Met in Pca cells (Fig. 3J). Correspondingly, Ki67 and CD44 showed a similar pattern to SOX9 (Fig. 3J). Taken together, HGF plays a central role in upregulating SOX9 in Pca cells and mediating the tumor-promoting effects of CAFs.

\section{HGF upregulates SOX9 expression via MEK1/2-ERK1/2 pathway}

Previous studies have reported that HGF induced dimerization and phosphorylation of c-Met, resulting in the activation of several downstream oncogenic signaling pathways including RAS/MAPK, PI3K/AKT and STAT3 [34]. To specify which pathway was involved in the regulation of SOX9 expression by HGF, we tested the total expression and phosphorylation level of representative proteins of RAS/MAPK, PI3K/AKT and STAT3 pathways in DU145 cells treated with $30 \mathrm{ng} / \mathrm{ml} \mathrm{HGF}$, by western blotting. It turned out that the phosphorylation of ERK1/2 (Thr202/Tyr204) was most enhanced in cells treated with HGF, while phosphorylation of JNK (Thr183/Tyr185) was increased slightly and others including p38, Akt, Stat3 and NF-KB remained unaffected (Fig. 4A). In a time course of HGF treatment, phosphorylation of ERK1/2 in both DU145 and 22Rv1 cells started rising as early as 10 mins upon stimulation and lasted for at least 2 hours (Fig. 4B). Further analysis revealed that phosphorylation of ERK1/2 by HGF was dose-dependent, and $30 \mathrm{ng} / \mathrm{ml} \mathrm{HGF}$ induced a strikingly increased phosphorylation of ERK1/2, which could be abolished by capmatinib pre-treatment (Fig. 4C). As expected, CAF-CM also caused a remarkable enhancement of phosphorylation of ERK1/2 compared with the control and NF-CM treatment, which could be abolished by capmatinib (Fig. S8C,D). Then, MEK1/2 inhibitor U0126 (Selleck Chemicals, S1102, Boston, MA, USA) and ERK1/2 inhibitor SCH772984 (Selleck Chemicals, S7101) were adopted to block MEK1/2-ERK1/2 pathway, and we found that the upregulation of SOX9 induced by HGF or CAF-CM was obviously abrogated (Fig. 4D, Fig. S8E). In addition, when MEK1/2-ERK1/2 pathway was inhibited, it repressed the reinforced abilities of cell invasion and sphere formation induced by HGF (Fig. 4E-H). These results implied that MEK1/2-ERK1/2 pathway was responsible for the upregulation of SOX9 and the tumorpromoting effects by HGF secreted from CAFs.

\section{FRA1 is the downstream effector of MEK1/2-ERK1/2 signaling and transcriptionally upregulates SOX9}

Previous reports showed that HGF treatment of hepatocellular carcinoma cells resulted in enhancement of the phosphorylation and total expression levels of AP-1 proteins which then mediated HGF-induced effects [35-37]. Multiple heterodimers of the FOS and JUN family bind to the TPA-responsive element (TRE, 5'-TGAG/CTCA-3') sequence and transcriptionally upregulate target genes. Since previous research has demonstrated that putative AP-1 binding site was found in the promoter region of mouse Sox 9 gene [38], we deduced that AP-1 transcription factors may be the downstream mediator of activated MEK1/2ERK1/2 to upregulate SOX9 expression. In order to identify which AP1 proteins were specifically activated by HGF, we analyzed the protein expression of all the members of AP-1 family, including c-Jun, JunB, 
JunD, c-Fos, FosB, Delta FosB, FRA1 and FRA2, in DU145 cells stimulated with $30 \mathrm{ng} / \mathrm{ml} \mathrm{HGF}$. The results revealed an obviously increase of FRA1 protein level, while the other members remained nearly unaffected (Fig. 5A). Further investigation showed that HGF treatment induced remarkable phosphorylation (Ser265) and increase of total expression of FRA1, as early as 10 mins in DU145 cells and 30 mins in 22Rv1 cells after HGF exposure, indicating that the inducement of phosphorylation and upregulation of FRA1 protein was quite rapid (Fig. 5B). Similar to the patterns of phosphorylation of ERK1/2, phosphorylation and upregulation of FRA1 by HGF was also in a dose-dependent fashion, and the markedly phosphorylation and upregulated expression of FRA1 induced by $30 \mathrm{ng} / \mathrm{ml} \mathrm{HGF}$ could be restored by capmatinib to that observed in the control treatment (Fig. 5C). Correspondingly, CAF-CM induced higher upregulation of FRA1 protein expression as well as its phosphorylation level compared to NF-CM (Fig. S8F). When capmatinib was added along with CAF-CM, the levels of phos-FRA1 and total FRA1 expression were restored to levels in the control treatment (Fig. S8G). U0126 and SCH772984 were used to inhibit MEK1/2-ERK1/2 pathway, then both the phosphorylation and upregulation of FRA1 stimulated by HGF or CAF-CM were abolished obviously (Fig. 5D, Fig. S8H). These data suggested that FRA1 was a downstream transcription factor activated by CAF-secreted HGF through MEK1/2-ERK1/2 pathway.

To evaluate the hypothesis that FRA1 transcriptionally upregulate SOX9 expression, we transiently overexpressed FRA1 as well as FOS and JUN in DU145 cells (AR negative). Forced expression of FRA1, FOS and JUN all increased protein level of SOX9, but FRA1 had the most obvious upregulating effect (Fig. $5 E$, left). qRT-PCR also showed a similar outcome that FRA1 increased SOX9 mRNA level most efficiently (Fig. 5E, right). In another two AR positive Pca cell lines, 22Rv1 and LNCaP, and an immortalized prostate epithelial cell, RWPE-1, the expression of SOX9 also increased upon FRA1 overexpression (Fig. 5F, Fig. S10A), indicating that upregulation of SOX9 by FRA1 was AR-independent. When FRA1 was depleted by specific siRNA, the upregulation of SOX9 induced by HGF or CAF-CM was obviously abrogated (Fig. 5G, Fig. S8I). Interestingly, FRA1 knockdown also visibly decreased the phosphorylation level of c-Met (Tyr1234/1235), suggesting that a positive feedback loop may exist between the HGF/c-Met pathway and FRA1 expression (Fig. 5G, Fig. S8I). To investigate the transcriptional regulation of SOX9 by FRA1, we analyzed the sequence of human SOX9 promoter and one TRE sequence (-551 -545) was found (Fig. $5 \mathrm{H}$ ). ChIP assay was performed using specific FRA1 and phos-FRA1 (Ser265) antibodies in DU145 cells stably overexpressing FRA1, and the precipitated DNA was amplified by two sets of primers across TRE sequence. The results showed both FRA1 and phos-FRA1 (Ser265) could bind to the target segment more than normal rabbit IgG (Fig. 5I, J), which indicated FRA1 could transcriptionally upregulate SOX9 expression. Further investigation showed that in DU145 cells treated with HGF (Fig. 5K) or CAF-CM (Fig. S8J), co-localization of FRA1 and SOX9 within the nuclei was quite apparent. In addition, Pca cells with FRA1 overexpressed formed larger xenograft tumors in mice, compared with the control group (Fig. S9). These data collectively indicated that FRA1 was the downstream effector of MEK1/2-ERK1/2 activated by HGF and transcriptionally mediated SOX9 upregulation.

\section{HGF/c-Met-FRA1-SOX9 axis is validated in mouse Pca cells and TCGA database}


On the one hand, mouse Hgf shares remarkable homology with human HGF at both mRNA (percent identity: about $88 \%$ ) and protein (percent identity: higher than $90 \%$ ) levels, and human and murine $\mathrm{HGF} / \mathrm{Hgf}$ are cross-reactive. On the other hand, TRE sequences were found in the promoter region of both human and mouse SOX9/Sox9 gene (Fig. 5H, Fig. 6E). In addition, binding site B (-600 bp $593 \mathrm{bp}$ ) in the mouse Sox9 promoter was found conserved with that found in the human SOX9 promoter $(-551 \sim$ -545). Therefore, we assumed that Hgf/c-Met-Erk1/2-Fra1 signaling was also responsible for the upregulation of Sox9 in mouse. Western blotting was carried out in a mouse Pca cell line, RM-1, and the results showed that recombinant murine Hgf (Peprotech, 315-23) also promoted Sox9 expression through c-Met-Erk1/2-Fra1 axis (Fig. 6A-D). ChIP assay with antibody against phos-Fra1 (Ser265) was performed in RM-1 cells treated with $30 \mathrm{ng} / \mathrm{ml} \mathrm{Hgf}$ for 6 hours, and it turned out that the activated Fra 1 could bind to all the three TRE sequence located in the promoter of Sox9 gene (Fig. 6E-G). We also exploited TCGA database and found that Pearson correlation analysis showed significant positive correlation between MET and SOX9 (Pearson $\mathrm{r}=0.268, p<0.0001, \mathrm{n}=499$, Fig.6H), MET and FRA1 (Pearson $\mathrm{r}=0.268, p$ $<0.0001, \mathrm{n}=499$, Fig.6I), FRA1 and SOX9 (Pearson $\mathrm{r}=0.312, p<0.0001, \mathrm{n}=499$, Fig.6J). These results strongly suggested that SOX9 expression regulated by HGF/c-Met-ERK1/2-FRA1 signaling was a relatively common mechanism in Pca cells.

\section{Discussion}

The transcription factor SOX9 is a critical regulator involved in prostate development, and reactivated in Pca initiation and progression [5-9]. In the current study, we validated the relation between SOX9 and Pca aggressiveness. SOX9 is positively corrected with Gleason scores and tumor staging of Pca. In addition, it promotes tumor growth, migration, invasion and cancer cell stemness both in vitro and in vivo. Since Pca with higher SOX9 is more aggressive with worse prognosis, it's urgently needed to uncover the key mechanism underlying SOX9 upregulation in Pca progression. Although previous study has revealed that in AR-positive Pca cells with TMPRSS2:ERG gene fusions, SOX9 expression could be upregulated by cooperation of ERG and AR [9], how SOX9 is elevated in AR-negative or TMPRSS2:ERG fusion-negative Pca cells still remains elusive.

CAFs is the main type of stromal cells in TME, providing a supportive microenvironment to induce and maintain more aggressive biological behaviors of cancer cells [39]. Increasing evidences support that CAFs promote tumorigenesis and progression of various cancers including Pca via the secretion of multiple paracrine factors including TGF $\beta 1$, IL6, HGF, EGF, VEGF, and FGF families [39, 40]. Meanwhile, previous studies showed that several kinds of cytokines such as FGFs, TGF $\beta 1$, and BMPs, induced the upregulation of SOX9 [13-18]. On the basis of these results, we hypothesized that the paracrine effects of CAFs might be involved in upregulating SOX9 in Pca cells. In fact, in vitro model of paracrine interaction between CAFs and Pca cells and in vivo experiments in our study demonstrated that SOX9 expression in Pca cells could indeed be upregulated by the paracrine effects of CAFs, both in AR-positive and AR-negative Pca cells (22Rv1 and DU145, respectively), suggesting an undiscovered mechanism underlying SOX9 elevation in Pca cells regardless of AR status. 
HGF is well known as a growth factor which possesses broad biological significance. Emerging evidences have demonstrated that HGF/c-Met plays a crucial role in tumorigenesis and cancer progression [41]. In our study, we identified that HGF was the most highly secreted factors by CAFs in TME compared with NFs and Pca cells. Furthermore, HGF was proved to be the key contributor for the upregulation of SOX9 in Pca cells and the tumor progression promoted by CAFs. Since the clinical significance of HGF/c-Met in Pca has been recognized, several agents targeting HGF/c-Met signaling including cabozantinib, foretinib, crizotinib, and rilotumumab underwent clinical trials [42]. In spite of the strong anti-tumor effects in pre-clinical studies, therapeutic agents targeting HGF/c-Met did not provide significant survival benefits for Pca patients in clinical trials [43]. One possible explanation for this seemingly inconsistent result is that enrolled patients in most clinical trials were not highly-selected. Patients without activated HGF/c-Met may get very limited benefits from HGF/c-Met inhibition agents. Rhee et al. found that the half-life of phosphorylated c-Met (Tyr1234/1235) is so short as less than 10 min that no reliable detection of the phosphorylation status of c-Met could be performed in human specimens [37]. Since the main challenge facing the efficacy of HGF/c-Met inhibition for Pca treatment is optimal patients enrollment, while capturing the activation status of HGF/c-Met signaling via detecting phos-c-Met by IHC staining in Pca tissues is quite unpractical, effective markers substituted for activation of HGF/c-Met signaling are urgently needed. Our work revealed that the growth of xenograft tumor with high SOX9 expression upregulated by CAFs could be effectively inhibited by capmatinib (Fig. $3 \mathrm{H}-\mathrm{J})$, and in vitro experiments indicated that HGF induced relatively continuous upregulation of SOX9 in Pca cells (Fig. 3F). Overall, SOX9 may serve as an alternative marker for the activated HGF/c-Met signaling to enroll the optimal Pca patients for HGF/c-Met inhibition treatment. Most importantly, SOX9 is much easier to detect, making it an ideal biomarker to predict treatment response of HGF/c-Met inhibition.

The binding of HGF to its receptor c-Met, triggers the activation of multiple downstream pathways including RAS/MAPK, PI3K/AKT, and STAT3 signals, then contributes to proliferation, metastasis, intrinsic or acquired drug resistance and tumor-initiating properties of cancer cells [34]. In this study, we identified MEK1/2-ERK1/2 was the bridge pathway activated by HGF/C-Met signaling, then took responsible for the activation of FRA1 and SOX9 elevation, suggesting that targeting MEK1/2-ERK1/2 pathway in combination with HGF/c-Met targeted therapy may be a more potential therapeutic strategy.

FRA1 is a member of the FOS family which binds to the TRE sequence and transcriptionally upregulate target genes. In our study, HGF treatment of Pca cells specifically upregulated FRA1 expression instead of other AP-1 family members (Fig. 5A). Further analysis showed that FRA1 was the crucial downstream effector of HGF/c-Met-ERK1/2-mediated SOX9 upregulation by directly binding to the TRE sequence in the promoter of SOX9 gene (Fig. 5H-J, Fig. 6E-G). Intriguingly, FRA1 interfering by siRNA also caused decrease of c-Met phosphorylation level (Fig. 5G, Fig. S8I), suggesting a positive regulatory circuit existed between HGF/c-Met signaling and FRA1 expression. In addition, SOX9 depletion by siRNA also significantly reduced the mRNA expression of FRA1, FRA2 and JUN (Fig. S10B), also indicating an interplay between SOX9 and AP-1 complex, which remains to be further elucidated. 


\section{Conclusions}

In conclusion, SOX9 is upregulated by CAF-secreted HGF through HGF/c-Met-ERK1/2-FRA1 axis both in AR-positive and AR-negative Pca cells, and mediates the tumor-promoting effects of CAFs (Fig. 6K). Better understanding of the molecular background of SOX9 in Pca provides a practical guide for the therapeutic applications involving HGF/c-Met targeted therapy.

\section{Abbreviations}

Pca

prostate cancer

TME

tumor microenvironment

CAFs

cancer-associated fibroblasts

NFs

normal fibroblasts

CAF-CM

conditional medium from Pca CAFs

NF-CM

conditional medium from NFs

TCGA

The Cancer Genome Atlas

HGF

hepatocyte growth factor

TRE

TPA-responsive element

SOX

Sry-related high mobility group box

AR

androgen receptor

ATCC

the American Type Culture Collect

IHC

immunohistochemistry

\section{Declarations}

Ethics approval and consent to participate 
The study was conducted in accordance with the Declaration of Helsinki principles. It was approved by the Ethics Committee of Drum Tower Hospital, Medical School of Nanjing University.

\section{Consent for publication}

All authors have read and consented to publication of the paper.

\section{Availability of data and materials}

The data and materials used and analyzed during the current study are available from the corresponding author on reasonable request.

\section{Competing interests}

The authors declare that they have no competing interests.

\section{Funding}

This study was supported by grants from the National Natural Science Foundation of China (81972388, 81802535, 81772710, 81602232), Nanjing Medical Science and Technique Development Foundation (QRX17128), Nanjing Health Distinguished Youth Fund (JQX16025), the Project of Invigorating Health Care through Science, Technology and Education, Jiangsu Provincial Key Medical Discipline (Laboratory) (ZDXKB2016014).

\section{Authors' contributions}

H Guo and X Qiu designed the study. H Qin, B Jiang and H Ma isolated the primary prostate stromal fibroblasts and performed all the cell assays. H Qin, C Pan and W Cao performed IHC and IF on human and mouse tissues. $\mathrm{H}$ Qin, $Y$ Yang and $B$ Jiang performed the PCR, WB and ELISA experiments. H Qin, Z Zhang and $M$ Chen performed the in vivo experiments. $Y$ Yang, $J$ Gao and $X$ Zhao analyzed the data and performed the statistics. W Diao and M Ding analyzed and interpreted the data. H Qin, W Chen, X Qiu and H Guo wrote the manuscript. H Guo supervised the study.

\section{Acknowledgments}

Not applicable.

\section{Author information}

Haixiang Qin, Yang Yang, Bo Jiang contributed equally to this work.

\section{Affiliations}

Department of Urology, Drum Tower Hospital, Medical School of Nanjing University, Institute of Urology, Nanjing University, Nanjing, 210008, China. 
Haixiang Qin, Yang Yang, Bo Jiang, Wei Chen, Wenli Diao, Meng Ding, Wenmin Cao, Mengxia Chen, Jie Gao, Xiaozhi Zhao, Xuefeng Qiu, Hongqian Guo

Immunology and Reproduction Biology Laboratory, State Key Laboratory of Analytical Chemistry for Life Science, Medical School, Nanjing University, Nanjing, 210093, China.

Chun Pan

Jiangsu Key Laboratory of Molecular Medicine, Nanjing University, Nanjing, 210093, China.

Chun Pan

Department of Urology, The First Affiliated Hospital of Wannan Medical College, Wuhu, 241001, China.

Zhenxing Zhang

Department of Urology, Shaanxi People's Hospital, Xi'an, 710068, China.

Haoxing Ma

Corresponding authors

Correspondence to Xuefeng Qiu and Hongqian Guo.

\section{References}

1. Chaboissier MC, Kobayashi A, Vidal VI, Lutzkendorf S, van de Kant HJ, Wegner M, et al. Functional analysis of Sox8 and Sox9 during sex determination in the mouse. Development. 2004;131:1891901.

2. Akiyama H, Chaboissier MC, Martin JF, Schedl A, de Crombrugghe B. The transcription factor Sox9 has essential roles in successive steps of the chondrocyte differentiation pathway and is required for expression of Sox5 and Sox6. Genes Dev. 2002;16:2813-28.

3. Stolt CC, Lommes P, Sock E, Chaboissier MC, Schedl A, Wegner M. The Sox 9 transcription factor determines glial fate choice in the developing spinal cord. Genes Dev. 2003;17:1677-89.

4. Akiyama H, Chaboissier MC, Behringer RR, Rowitch DH, Schedl A, Epstein JA, et al. Essential role of Sox9 in the pathway that controls formation of cardiac valves and septa. Proc Natl Acad Sci USA. 2004;101:6502-7.

5. Thomsen MK, Francis JC, Swain A. The role of Sox9 in prostate development. Differ Res Biol Divers. 2008;76:728-35.

6. Huang Z, Hurley PJ, Simons BW, Marchionni L, Berman DM, Ross AE, et al. Sox9 is required for prostate development and prostate cancer initiation. Oncotarget. 2012;3:651-63. 
7. Wang H, Leav I, Ibaragi S, Wegner M, Hu GF, Lu ML, et al. SOX9 is expressed in human fetal prostate epithelium and enhances prostate cancer invasion. Cancer research. 2008;68:1625-30.

8. Thomsen MK, Ambroisine L, Wynn S, Cheah KS, Foster CS, Fisher G, et al. SOX9 elevation in the prostate promotes proliferation and cooperates with PTEN loss to drive tumor formation. Cancer research. 2010;70:979-87.

9. Cai C, Wang H, He HH, Chen S, He L, Ma F, et al. ERG induces androgen receptor-mediated regulation of SOX9 in prostate cancer. J Clin Investig. 2013;123:1109-22.

10. Wang H, McKnight NC, Zhang T, Lu ML, Balk SP, Yuan X. SOX9 is expressed in normal prostate basal cells and regulates androgen receptor expression in prostate cancer cells. Cancer research. 2007;67:528-36.

11. Xie Y, Lu W, Liu S, Yang Q, Carver BS, Li E, et al. Crosstalk between nuclear MET and SOX9/betacatenin correlates with castration-resistant prostate cancer. Molecular endocrinology (Baltimore Md). 2014;28:1629-39.

12. Chen X, Li Q, Liu X, Liu C, Liu R, Rycaj K, et al. Defining a Population of Stem-like Human Prostate Cancer Cells That Can Generate and Propagate Castration-Resistant Prostate Cancer. Clinical cancer research: an official journal of the American Association for Cancer Research. 2016;22:4505-16.

13. Nguyen MB, Cohen I, Kumar V, Xu Z, Bar C, Dauber-Decker KL, et al. FGF signalling controls the specification of hair placode-derived SOX9 positive progenitors to Merkel cells. Nature communications. 2018;9:2333.

14. Chatzeli L, Gaete M, Tucker AS. Fgf10 and Sox9 are essential for the establishment of distal progenitor cells during mouse salivary gland development. Development. 2017;144:2294-305.

15. Li T, Huang H, Shi G, Zhao L, Li T, Zhang Z, et al. TGF-beta1-SOX9 axis-inducible COL10A1 promotes invasion and metastasis in gastric cancer via epithelial-to-mesenchymal transition. Cell death disease. 2018;9:849.

16. Wu C, Jiao H, Lai Y, Zheng W, Chen K, Qu H, et al. Kindlin-2 controls TGF-beta signalling and Sox9 expression to regulate chondrogenesis. Nature communications. 2015;6:7531.

17. Kishi S, Abe H, Akiyama H, Tominaga T, Murakami T, Mima A, et al. SOX9 protein induces a chondrogenic phenotype of mesangial cells and contributes to advanced diabetic nephropathy. $J$ Biol Chem. 2011;286:32162-9.

18. Raspopovic J, Marcon L, Russo L, Sharpe J. Modeling digits. Digit patterning is controlled by a BmpSox9-Wnt Turing network modulated by morphogen gradients. 345. New York: Science; 2014. pp. 566-70.

19. Giannoni E, Bianchini F, Masieri L, Serni S, Torre E, Calorini L, et al. Reciprocal activation of prostate cancer cells and cancer-associated fibroblasts stimulates epithelial-mesenchymal transition and cancer stemness. Cancer research. 2010;70:6945-56.

20. Liao CP, Adisetiyo H, Liang M, Roy-Burman P. Cancer-associated fibroblasts enhance the glandforming capability of prostate cancer stem cells. Cancer research. 2010;70:7294-303. 
21. Mishra R, Haldar S, Placencio V, Madhav A, Rohena-Rivera K, Agarwal P, et al. Stromal epigenetic alterations drive metabolic and neuroendocrine prostate cancer reprogramming. J Clin Investig. 2018;128:4472-84.

22. Kato M, Placencio-Hickok VR, Madhav A, Haldar S, Tripathi M, Billet S, et al. Heterogeneous cancerassociated fibroblast population potentiates neuroendocrine differentiation and castrate resistance in a CD105-dependent manner. Oncogene. 2019;38:716-30.

23. Ippolito L, Morandi A, Taddei ML, Parri M, Comito G, Iscaro A, et al. Cancer-associated fibroblasts promote prostate cancer malignancy via metabolic rewiring and mitochondrial transfer. Oncogene. 2019;38:5339-55.

24. Cunha GR, Hayward SW, Wang YZ, Ricke WA. Role of the stromal microenvironment in carcinogenesis of the prostate. International journal of cancer. 2003;107:1-10.

25. Kramer N, Schmollerl J, Unger C, Nivarthi H, Rudisch A, Unterleuthner D, et al. Autocrine WNT2 signaling in fibroblasts promotes colorectal cancer progression. Oncogene. 2017;36:5460-72.

26. Valenti G, Quinn HM, Heynen G, Lan L, Holland JD, Vogel R, et al. Cancer Stem Cells Regulate CancerAssociated Fibroblasts via Activation of Hedgehog Signaling in Mammary Gland Tumors. Cancer research. 2017;77:2134-47.

27. Liu C, Liu L, Chen X, Cheng J, Zhang H, Zhang C, et al. LSD1 Stimulates Cancer-Associated Fibroblasts to Drive Notch3-Dependent Self-Renewal of Liver Cancer Stem-like Cells. Cancer research. 2018;78:938-49.

28. Vidal VP, Chaboissier MC, Lutzkendorf S, Cotsarelis G, Mill P, Hui CC, et al. Sox9 is essential for outer root sheath differentiation and the formation of the hair stem cell compartment. Current biology: $\mathrm{CB}$. 2005;15:1340-51.

29. Blache $P$, van de Wetering M, Duluc I, Domon C, Berta P, Freund JN, et al. SOX9 is an intestine crypt transcription factor, is regulated by the Wnt pathway, and represses the CDX2 and MUC2 genes. $J$ Cell Biol. 2004;166:37-47.

30. Song S, Ajani JA, Honjo S, Maru DM, Chen Q, Scott AW, et al. Hippo coactivator YAP1 upregulates SOX9 and endows esophageal cancer cells with stem-like properties. Cancer research. 2014;74:4170-82.

31. Ma F, Ye H, He HH, Gerrin SJ, Chen S, Tanenbaum BA, et al. SOX9 drives WNT pathway activation in prostate cancer. J Clin Investig. 2016;126:1745-58.

32. Steffan JJ, Williams BC, Welbourne T, Cardelli JA. HGF-induced invasion by prostate tumor cells requires anterograde lysosome trafficking and activity of $\mathrm{Na}+-\mathrm{H}+$ exchangers. Journal of cell science. 2010;123:1151-9.

33. Jensen AR, David SY, Liao C, Dai J, Keller ET, Al-Ahmadie H, et al. Fyn is downstream of the HGF/MET signaling axis and affects cellular shape and tropism in PC3 cells. Clinical cancer research: an official journal of the American Association for Cancer Research. 2011;17:3112-22.

34. Bradley CA, Salto-Tellez M, Laurent-Puig P, Bardelli A, Rolfo C, Tabernero J, et al. Targeting c-MET in gastrointestinal tumours: rationale, opportunities and challenges. Nature reviews Clinical oncology. 
2017;14:562-76.

35. Seol DW, Chen Q, Zarnegar R. Transcriptional activation of the hepatocyte growth factor receptor (cmet) gene by its ligand (hepatocyte growth factor) is mediated through AP-1. Oncogene. 2000;19:1132-7.

36. Lau EY, Lo J, Cheng BY, Ma MK, Lee JM, Ng JK, et al. Cancer-Associated Fibroblasts Regulate TumorInitiating Cell Plasticity in Hepatocellular Carcinoma through c-Met/FRA1/HEY1 Signaling. Cell reports. 2016;15:1175-89.

37. Rhee H, Kim HY, Choi JH, Woo HG, Yoo JE, Nahm JH, et al. Keratin 19 Expression in Hepatocellular Carcinoma Is Regulated by Fibroblast-Derived HGF via a MET-ERK1/2-AP1 and SP1 Axis. Cancer research. 2018;78:1619-31.

38. He Y, Zhu W, Shin MH, Gary J, Liu C, Dubois W, et al. cFOS-SOX9 Axis Reprograms Bone MarrowDerived Mesenchymal Stem Cells into Chondroblastic Osteosarcoma. Stem cell reports. 2017;8:1630-44.

39. Gascard P, TIsty TD. Carcinoma-associated fibroblasts: orchestrating the composition of malignancy. Genes Dev. 2016;30:1002-19.

40. Chen $X$, Song E. Turning foes to friends: targeting cancer-associated fibroblasts. Nature reviews Drug discovery. 2019;18:99-115.

41. Gherardi E, Birchmeier W, Birchmeier C, Vande Woude G. Targeting MET in cancer: rationale and progress. Nature reviews Cancer. 2012;12:89-103.

42. Varkaris A, Corn PG, Gaur S, Dayyani F, Logothetis CJ, Gallick GE. The role of HGF/c-Met signaling in prostate cancer progression and c-Met inhibitors in clinical trials. Expert opinion on investigational drugs. 2011;20:1677-84.

43. Ryan CJ, Rosenthal M, Ng S, Alumkal J, Picus J, Gravis G, et al. Targeted MET inhibition in castrationresistant prostate cancer: a randomized phase II study and biomarker analysis with rilotumumab plus mitoxantrone and prednisone. Clinical cancer research: an official journal of the American Association for Cancer Research. 2013;19:215-24.

\section{Figures}


A

Normal

$3+3=6$

$3+4=7$

$4+3=7$

$4+4=8$

$4+5=9$
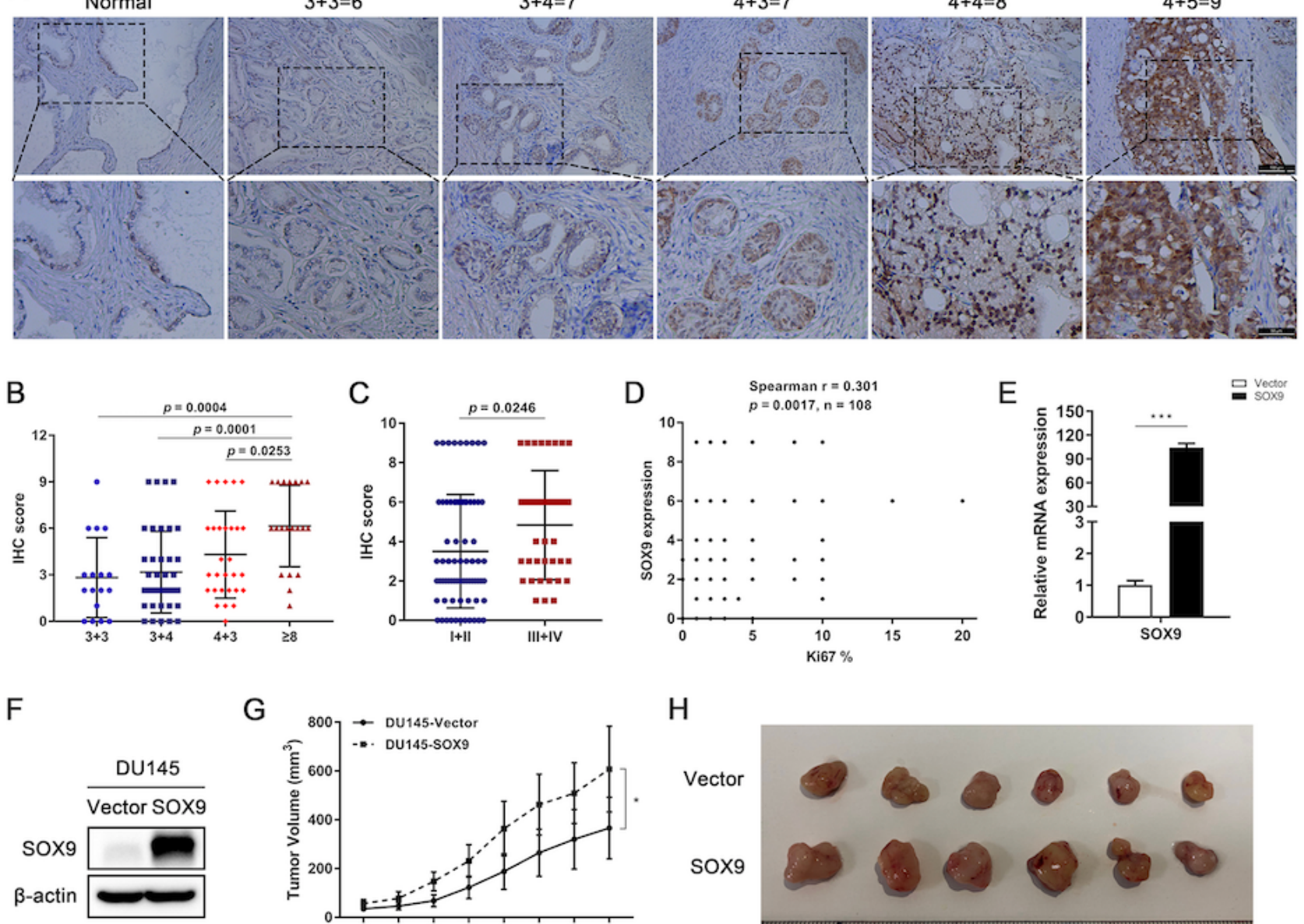

G

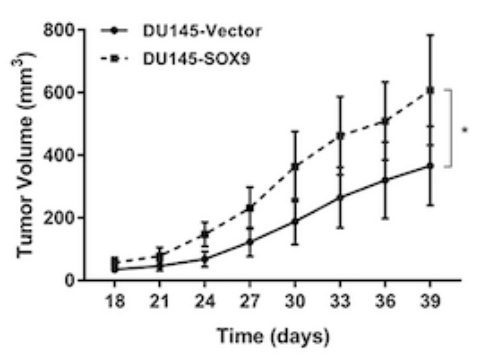

$\mathrm{H}$
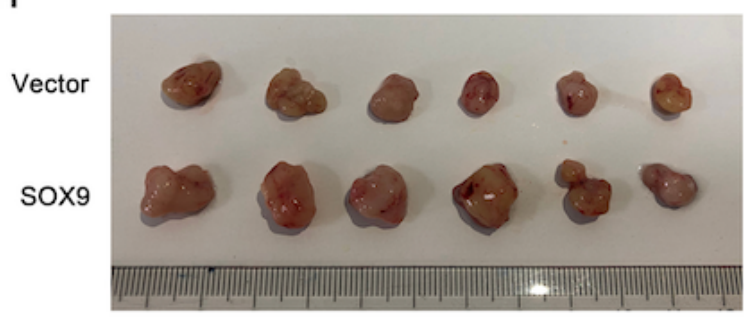

I

HE

sox9

Ki67

CD44

CD133
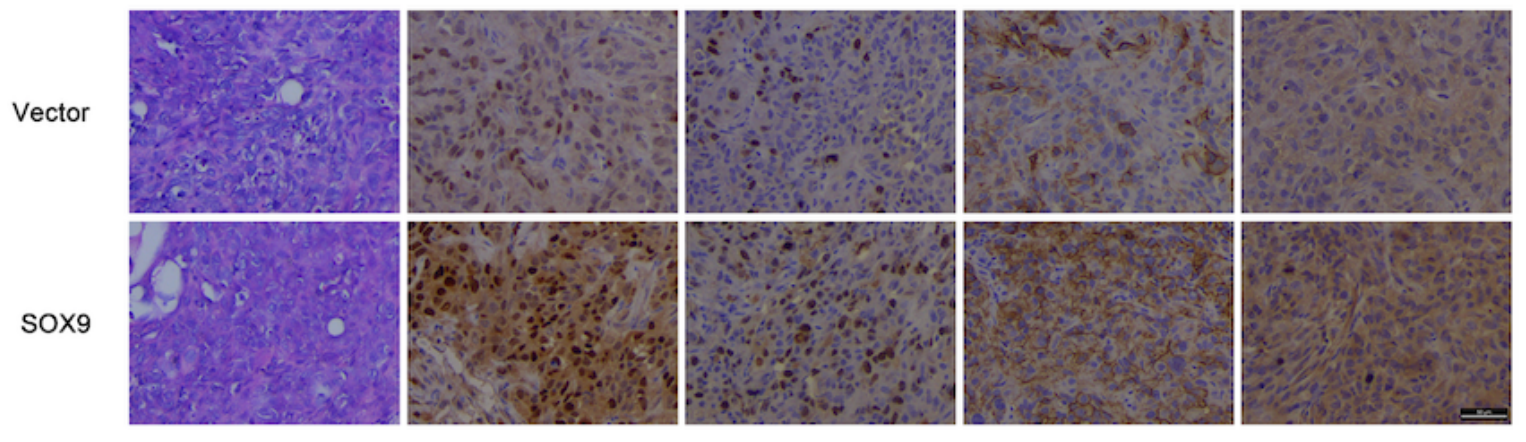

Figure 1

SOX9 elevation in Pca is positively correlated with cancer aggressiveness. (A) Representative images of SOX9 IHC staining in normal prostate tissues and Pca specimens with different Gleason Scores $(n=108)$. Scale bars represent $100 \mu \mathrm{m}$ (upper panel) and $50 \mu \mathrm{m}$ (lower panel). (B, C) IHC scores of SOX9 expression in Pca tissues with Gleason Scores $3+3,3+4,4+3$ and $\geq 8$ (B), and with stages I+II and III+IV (C). (D) Spearman correlation between the percentage of Ki67-positive tumor cells and SOX9 expression 
in Pca tissues. (E, F) The efficiency of SOX9 overexpression in lentiviral infected DU145 cells was validated by qRT-PCR (E) and western blotting (F). (G) The growth rate of subcutaneous tumors formed of control or SOX9-overexpressing DU145 cells. (H) Photograph of dissected tumors in control or SOX9overexpressing groups. (I) Representative pictures of HE staining and IHC staining of SOX9, Ki67, CD44 and CD133 in the xenografts. Scale bars represent $50 \mu \mathrm{m}$. ${ }^{*} \mathrm{p}<0.05 ;{ }^{* \star} \mathrm{p}<0.01 ;{ }^{* \star *} \mathrm{p}<0.001$.

A

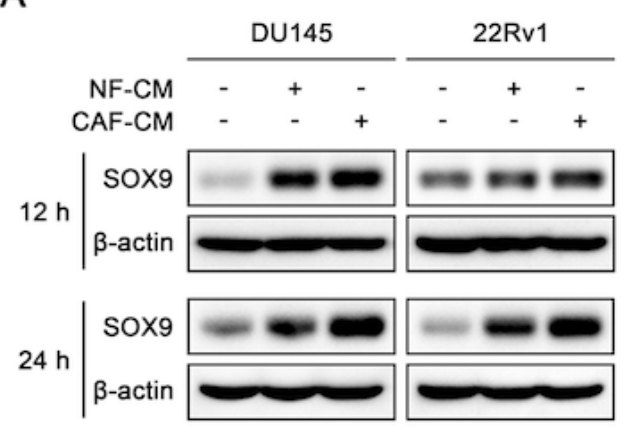

C

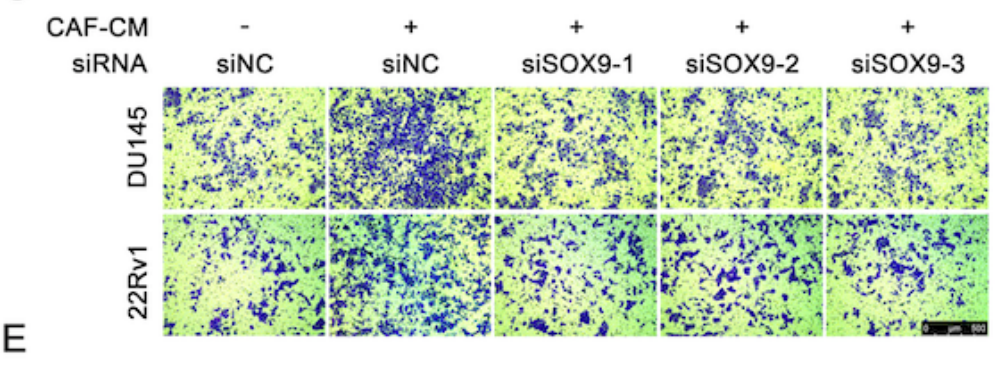
CAF-CM
SiRNA

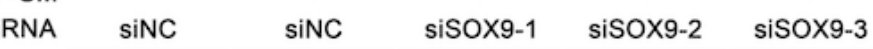
ำ 妾

$\mathrm{D}$
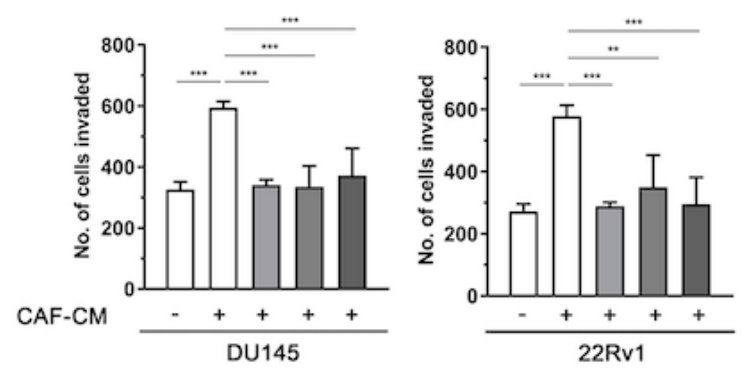

B
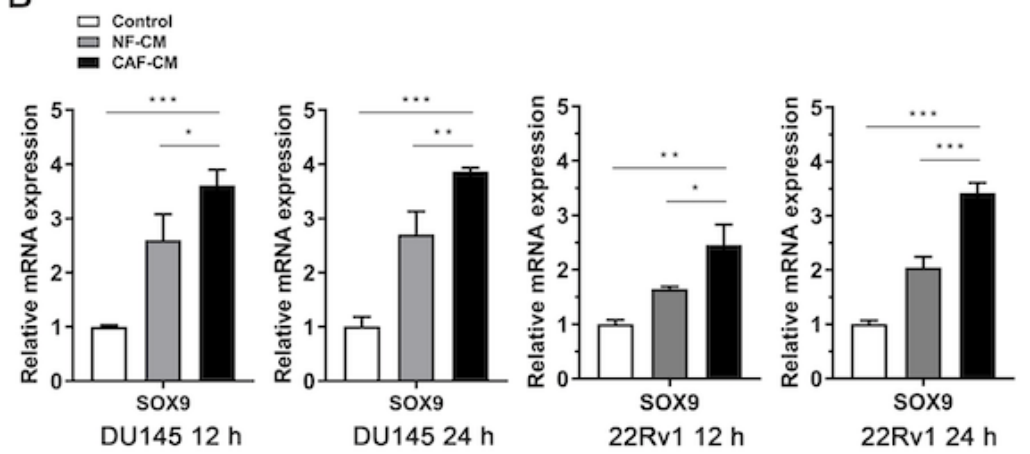

G
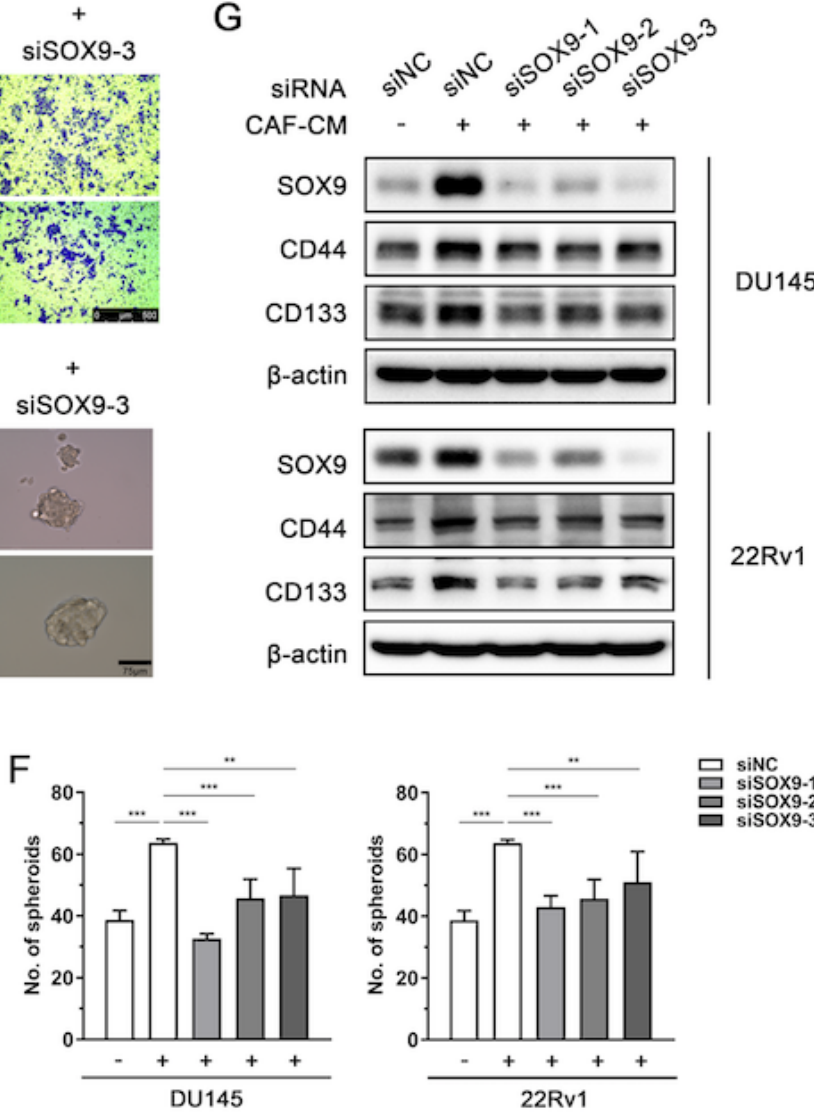

\section{Figure 2}

SOX9 in Pca is upregulated by paracrine factors from CAFs and essential for CAF-mediated tumor progression. (A, B) The protein level (A) and mRNA expression (B) of SOX9 in DU145 and 22Rv1 cells after treatment of control medium or NF-CM and CAF-CM for 12 and 24 hours. (C, D) RNA interfering of 
SOX9 abolished CAF-induced cell invasion of DU145 and 22Rv1 cells. (E, F) Knockdown of SOX9 obviously decreased the sphere size and number increased by CAF-CM. (G) Western blotting showed SOX9 depletion abrogated the enhanced expression of stemness markers (CD44, CD133) induced by CAF-CM. Each in vitro assay involving CAF-CM and NF-CM treatment was conducted by using at least three pairs of CAFs and NFs, and a representative result was presented in the figure. ${ }^{\star} p<0.05 ;{ }^{\star \star} p<0.01$; $\star \star \star p<0.001$.

A

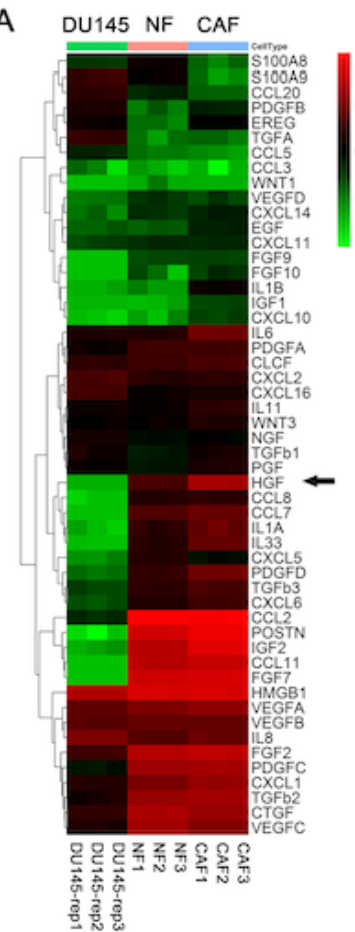

B

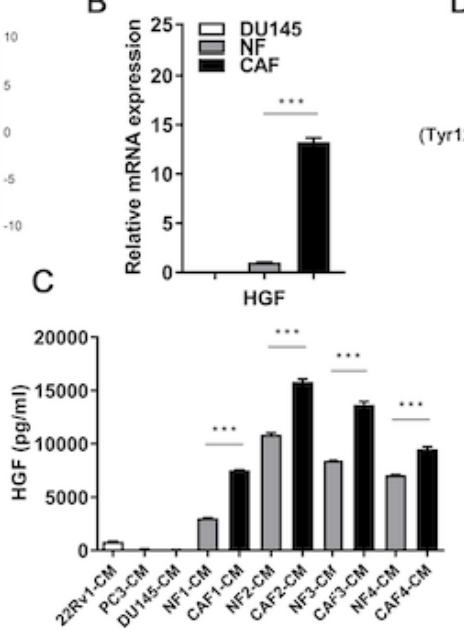

D

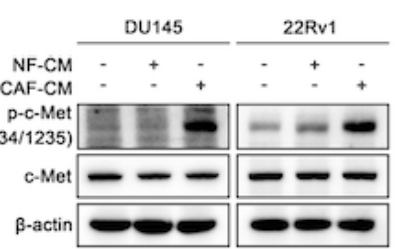

E

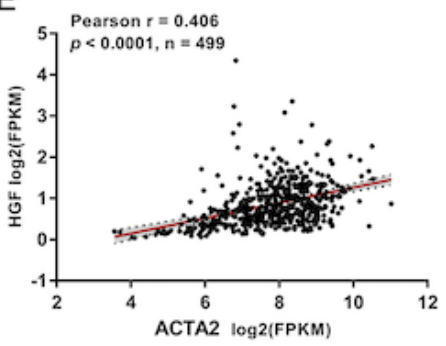

22Rv1

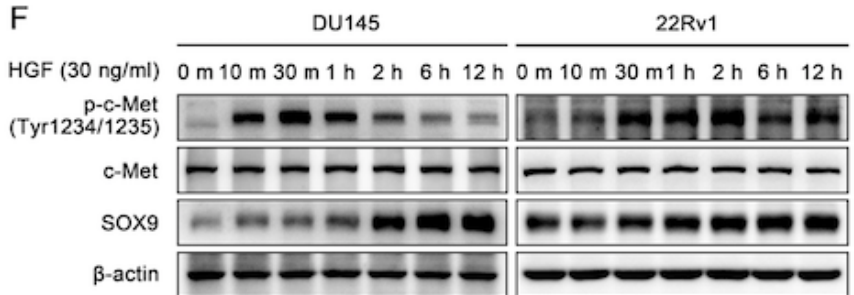

G
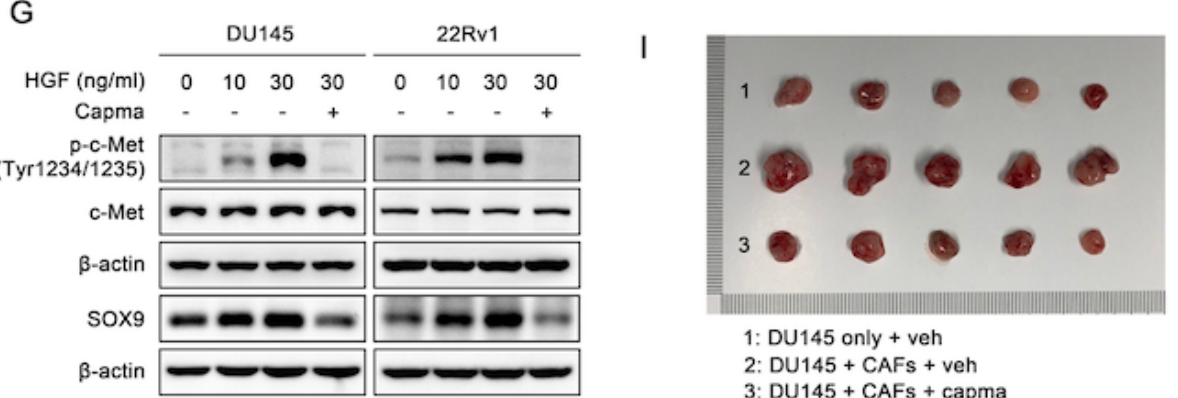

1: DU145 only + veh

2: DU145 + CAFs + veh

3: DU145 + CAFs + capma

$\mathrm{H}$

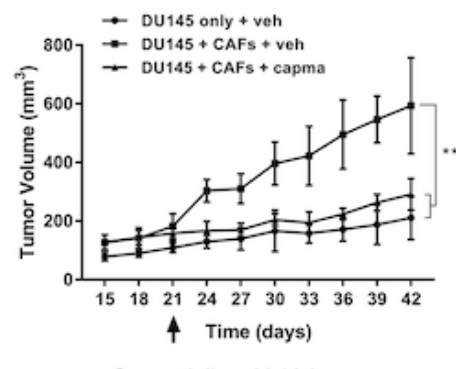

Capmatinib or Vehicle
J p-c-Met (Tyr1234/1235)

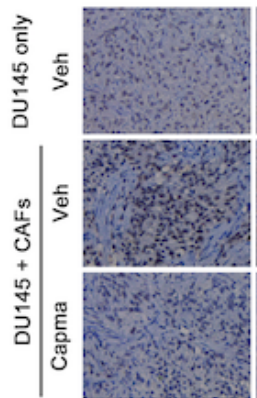

sox9

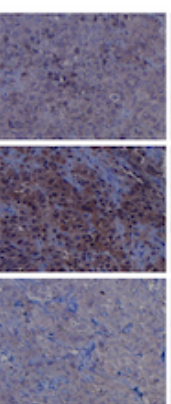

Ki67

CD44

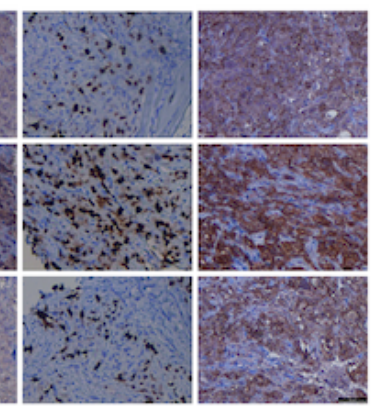


HGF secreted by CAFs upregulates SOX9 expression in Pca cells. (A) Hierarchical clustering and heatmap showed the differential expression patterns of 52 TME-related paracrine factors in DU145 cells and three pairs of NFs and CAFs, by a small scale qRT-PCR screening. A three-colored scale was used with red, black, and green indicating high, intermediate and low expression, respectively. (B) qRT-PCR validation of HGF mRNA expression in DU145 cells and isolated fibroblasts. (C) HGF concentration in conditional media of three Pca cell lines (22Rv1, PC3, DU145) and four pairs of NFs and CAFs was quantified by ELISA assay. (D) The phosphorylated c-Met (Tyr1234/1235) expression in DU145 and 22Rv1 cells treated with control medium, NF-CM and CAF-CM was measured by western blotting. (E) Pearson correlation between mRNA expression of ACTA2 and HGF in Pca data from TCGA. $(F, G)$ Western blotting showed that recombinant human HGF induced continuous SOX9 elevation in Pca cells (F) and upregulation of SOX9 by HGF was in a dose-dependent fashion $(G)$. $(H)$ The growth rate of subcutaneous xenografts formed of DU145 cells alone with vehicle treatment, and DU145 cells accompanied by equivalent CAFs with vehicle or capmatinib treatment (capmatinib, oral, $10 \mathrm{mg} / \mathrm{kg}$ body weight, 2 doses/day). (I) Dissected tumors collected in the end point from the three groups. (J) Representative images of IHC staining of phos-c-Met (Tyr1234/1235), SOX9, Ki67 and CD44 in the xenografts. Scale bars represent $50 \mu \mathrm{m}$. Each in vitro assay involving CAF-CM and NF-CM treatment was conducted by using at least three pairs of CAFs and NFs, and a representative result was presented in the figure. ${ }^{*} p<0.05 ;{ }^{* \star} p<0.01 ;{ }^{* \star} p<0.001$. 
A

B
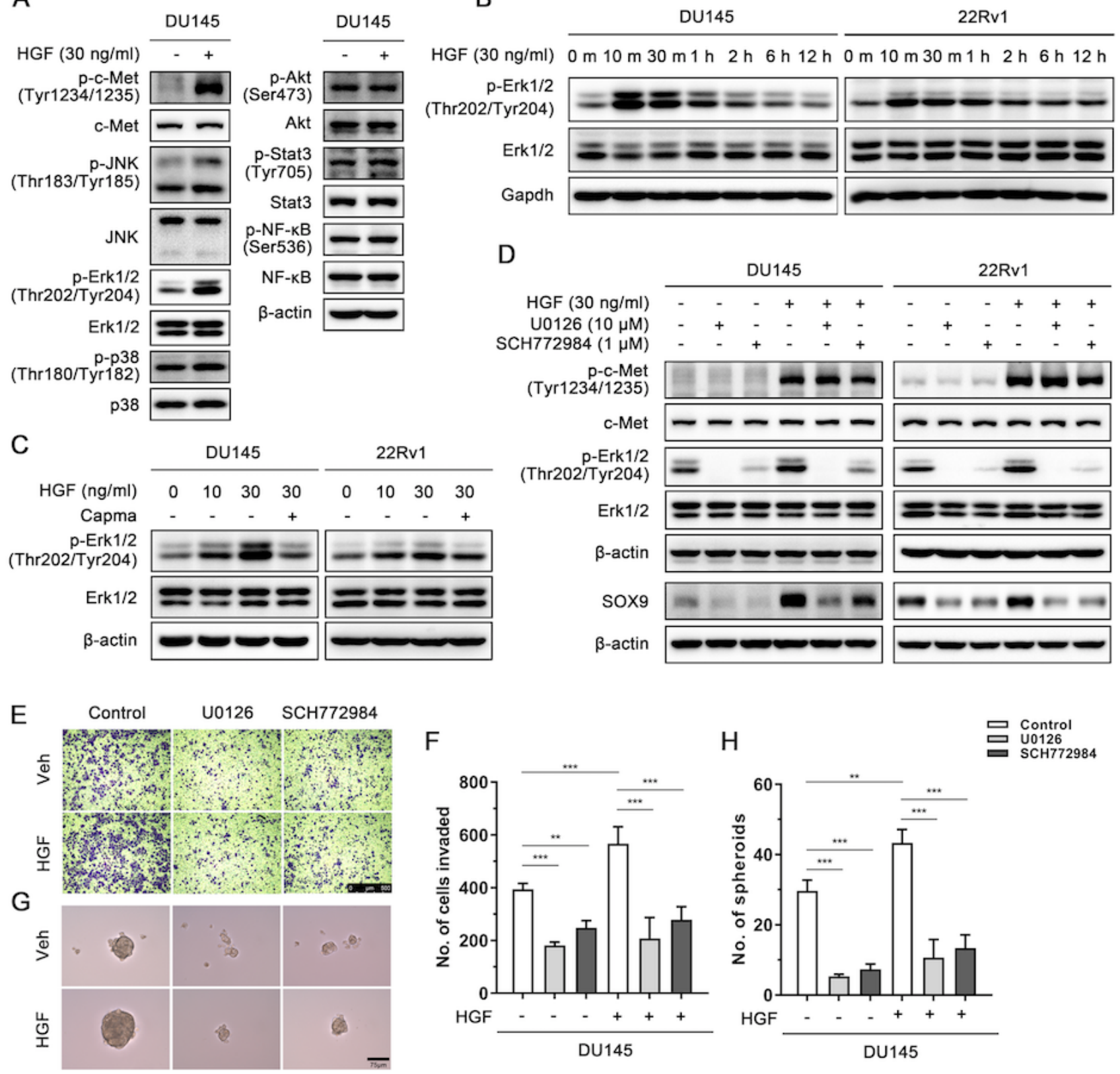

\section{Figure 4}

HGF upregulates SOX9 through activating MEK1/2-ERK1/2 pathway. (A) The activated and total expression of representative proteins of RAS/MAPK, PI3K/AKT and STAT3 pathways in DU145 cells treated with $30 \mathrm{ng} / \mathrm{ml}$ HGF were determined by western blotting. (B) Western blotting showed that phosphorylated ERK1/2 (Thr202/Tyr204) level in Pca cells started rising in 10 mins upon HGF stimulation and lasted for at least 2 hours. (C) Phosphorylated status of ERK1/2 induced by HGF was concentration-dependent, which could be abolished by capmatinib pretreatment. (D) Western blotting showed that combination of MEK1/2 inhibitor U0126 or ERK1/2 inhibitor SCH772984 with HGF 
observably restored the upregulation of SOX9 induced following HGF treatment. (E-H) Inhibition of MEK1/2-ERK1/2 pathway prominently abolished HGF-induced cell invasion (E, $F$ ) and the ability of sphere formation $(G, H) .{ }^{\star} p<0.05 ;{ }^{* \star} p<0.01 ;{ }^{* \star *} p<0.001$.

A

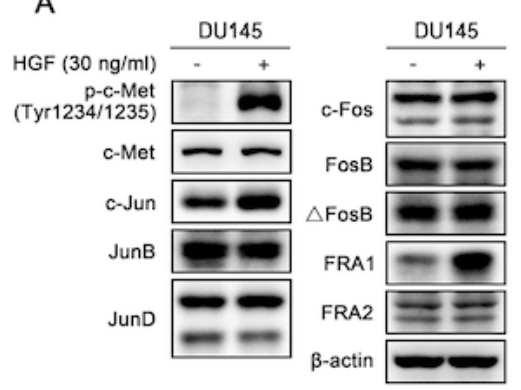

C

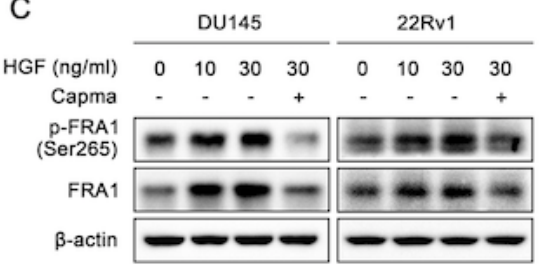

F

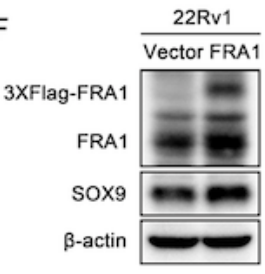

B
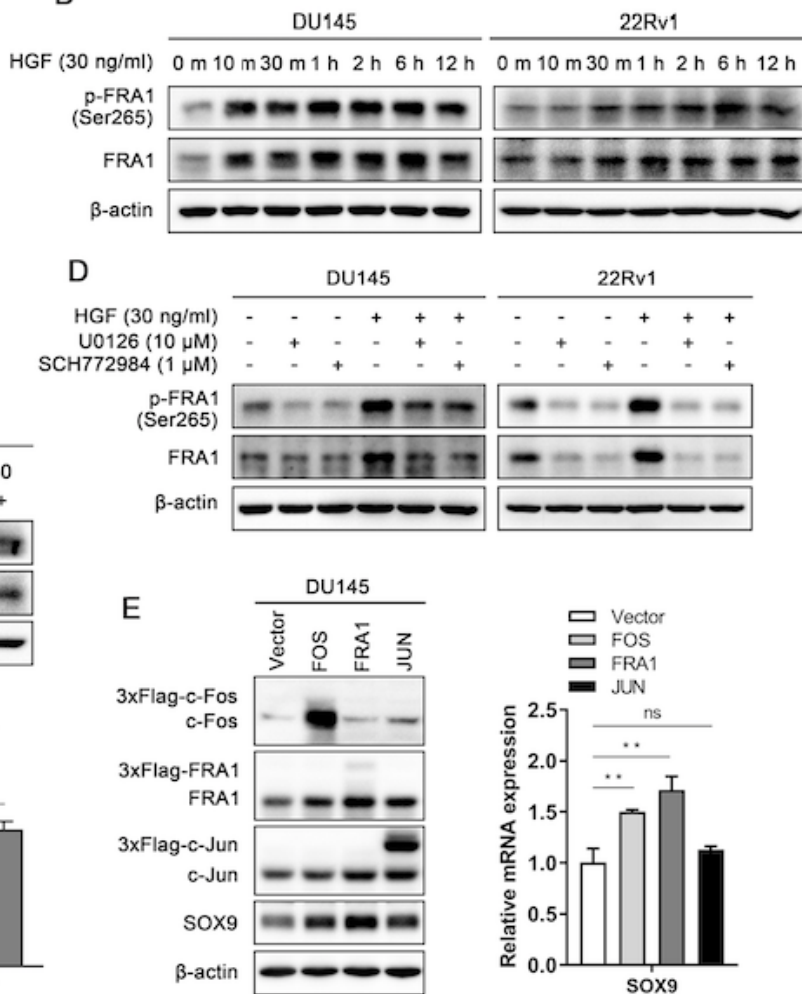
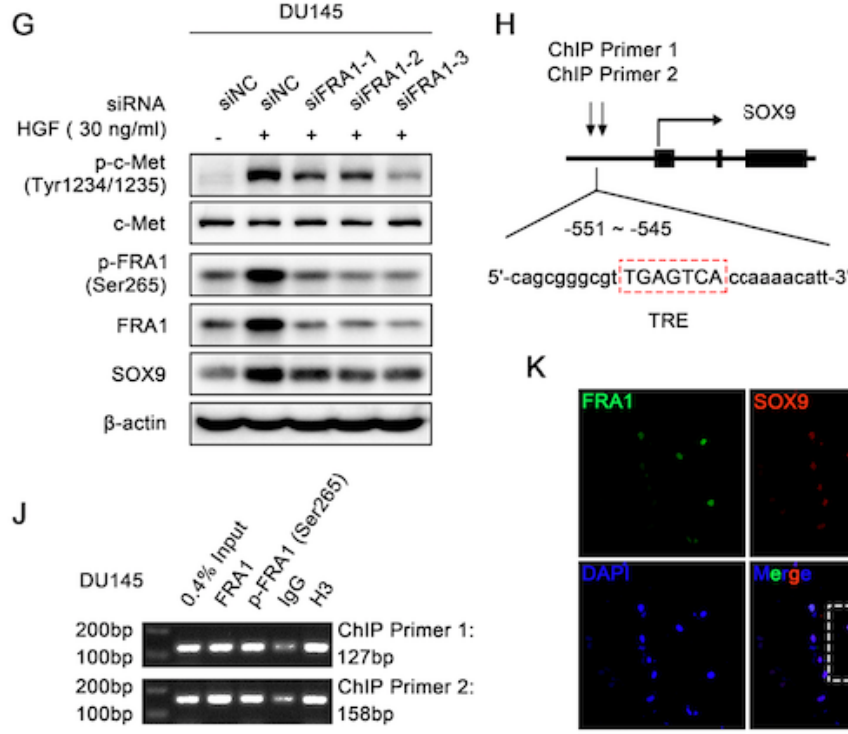

5'-cagcgggcgt TGAGTCA ccaaaacatt-3'

TRE

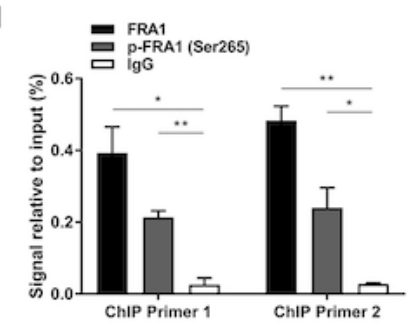

$\mathrm{K}$

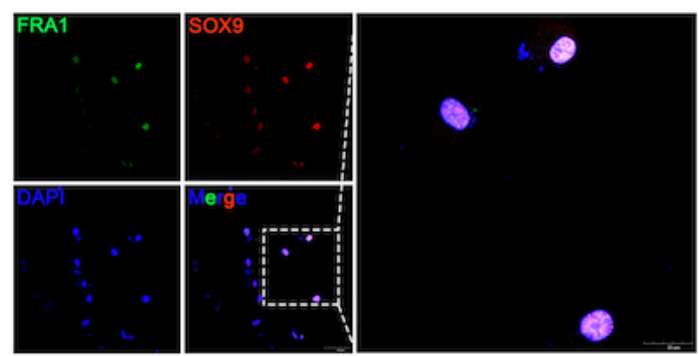

\section{Figure 5}

FRA1 is the downstream effector of HGF/c-Met-ERK1/2 signaling to transcriptionally upregulate SOX9. (A) The total protein levels of all the members of AP-1 family, including c-Jun, JunB, JunD, c-Fos, FosB, 
Delta FosB, FRA1 and FRA2, were detected by western blotting in DU145 cells treated with $30 \mathrm{ng} / \mathrm{ml} \mathrm{HGF}$. (B, C) Phosphorylated status (Ser265) and total expression of FRA1 were analyzed by western blotting in Pca cells with HGF treatment in a time course (B) or dose-escalating manner (C). (D) U0126 and SCH772984 signally abolished the increase of phos-FRA1 and FRA1 levels caused by HGF stimulation. (E) Protein expression (left) and relative mRNA expression (right) of SOX9 in DU145 cells transiently transfected with vector or FOS, FRA1, JUN plasmids. (F) Protein expression (left) and relative mRNA expression (right) of SOX9 in 22Rv1 cells transiently transfected with vector or FRA1 plasmids. $(G)$ Knockdown of FRA1 obviously abrogated the upregulation of SOX9 induced by HGF. (H) Schematic diagram showed the location of TRE sequence in the promoter region of SOX9 gene, and two sets of ChIP Primers were used to amplify the precipitated DNA(I, J) ChIP-qRT-PCR (I) and ChIP-PCR (J) showed both FRA1 and phos-FRA1 (Ser265) could bind to the segment around TRE sequence. Normal rabbit IgG was used as a negative control. (K) Confocal images of co-localization of FRA1 and SOX9 in DU145 cells treated with HGF. Scale bars represent $50 \mu \mathrm{m}$ (left panel) and $20 \mu \mathrm{m}$ (right panel), respectively. ${ }^{*}<<0.05$; $\star * \mathrm{p}<0.01 ; * \star * \mathrm{p}<0.001 ; \mathrm{ns}$, not significant. 
A

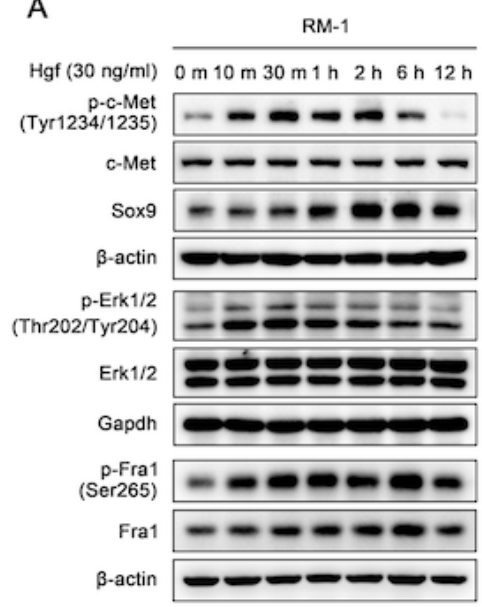

D

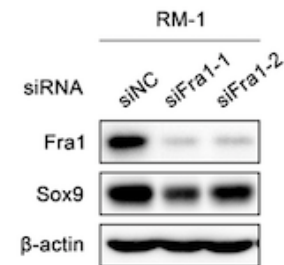

$\mathrm{F}$

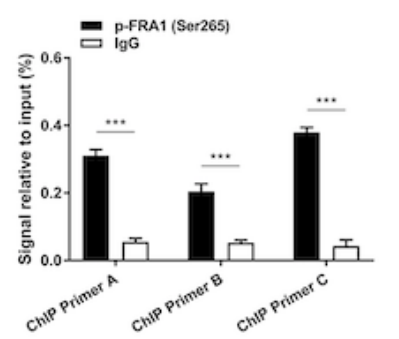

B

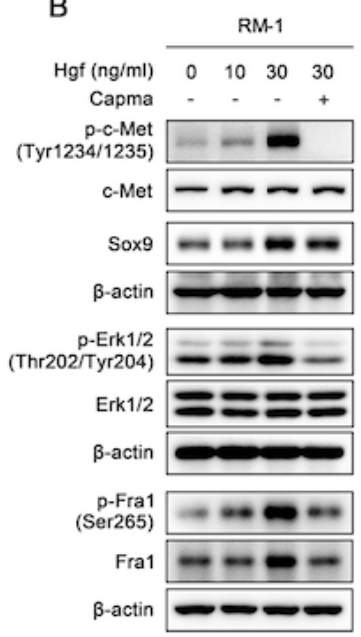

E

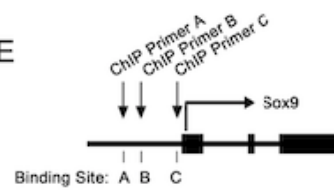

Binding Site A: -902 -895

5'-acggtgttg TGACTCA gtcaggagge-3.

Binding Site B: $-600 \sim-593$

5'-cagegggcgt TGAGTCAicceaaacatt-3'

Binding Site $C:-6 B \sim-61$

5'-ccaacactga TGACTCA agegctagcc- $3^{\prime}$

G

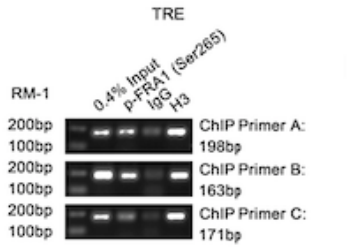

C

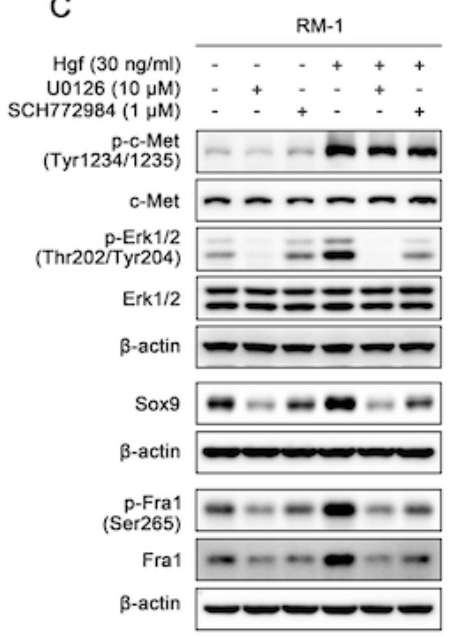

$\mathrm{H}$

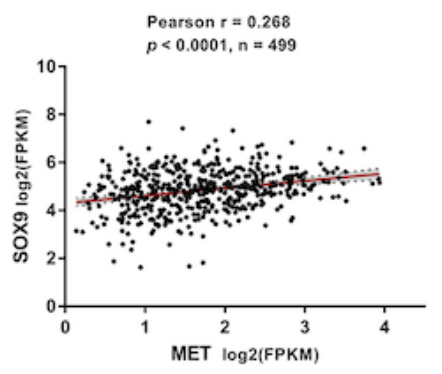

I

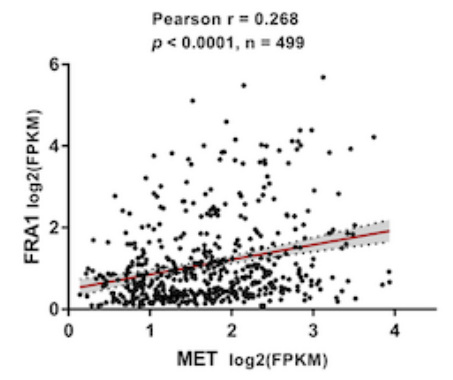

J
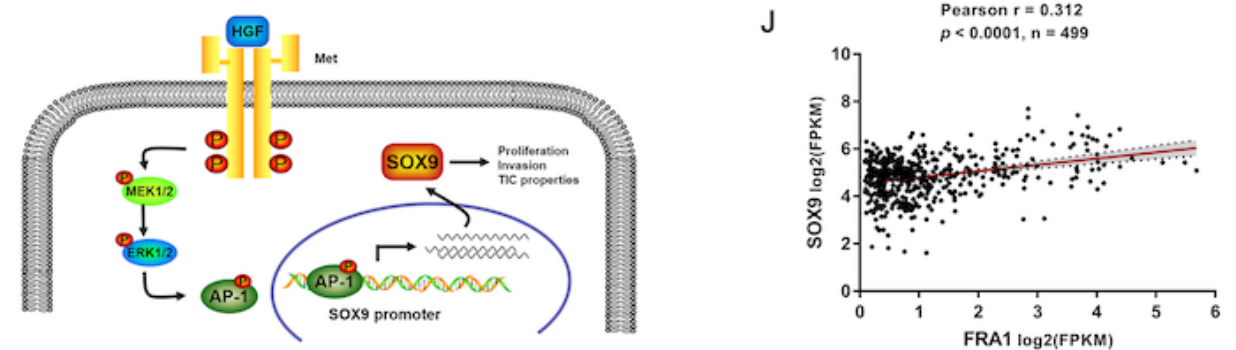

\section{Figure 6}

Validation of HGF/c-Met-FRA1-SOX9 axis in mouse Pca cells and Pca mRNA profiles from TCGA. (A) Western blotting showed that recombinant murine Hgf induced upregulation of Sox9 expression in a time-dependent fashion, as well as the expression levels of phos-Erk1/2 (Thr202/Tyr204), phos-Fra1 (Ser265) and Fra1, in the mouse Pca cell line, RM-1. (B) Increased expression of Sox9 following the treatment of $30 \mathrm{ng} / \mathrm{ml} \mathrm{Hgf}$ could be abrogated by c-Met targeted antagonist, capmatinib. Expression 
levels of phos-Erk1/2 (Thr202/Tyr204), phos-Fra1 (Ser265) and Fra1 presented a similar pattern to Sox9. (C) Inhibition of Mek1/2-Erk1/2 signaling by U0126 and SCH772984 decreased the Hgf-induced upregulation of Sox9, as well as phos-Fra1 (Ser265) and Fra1. (D) Protein expression of Sox9 in RM-1 cells transiently transfected with siRNA targeting Fra1. (E) Three TRE sequences were found in the promoter of Sox 9 gene, and binding site B (-600 bp -593 bp) was conserved among human and mouse. (F, G) ChIP assay indicated that phos-FRA1 (Ser265) could bind to all the three segments around TRE sequence. Results were normalized to the input. (H-J) Pearson correlation analysis between MET and SOX9 $(\mathrm{H})$, MET and FRA1 (I), FRA1 and SOX9 $(\mathrm{J})$ in Pca data from TCGA. (K) Schematic illustration of the regulatory mechanism underlying SOX9 upregulation in Pca cells by HGF derived from CAFs via HGF/cMet-ERK1/2-FRA1 axis. ${ }^{*} p<0.05 ;{ }^{* \star} p<0.01 ;{ }^{* \star *} p<0.001$.

\section{Supplementary Files}

This is a list of supplementary files associated with this preprint. Click to download.

- supplement10.docx 